\title{
WEIGHTED INEQUALITIES FOR BOCHNER-RIESZ MEANS IN THE PLANE
}

\author{
Anthony Carbery and Andreas Seeger
}

\section{Introduction}

For a Schwartz function $f \in \mathcal{S}\left(\mathbb{R}^{2}\right)$ let $\widehat{f}(\xi)=\int f(y) e^{-i\langle y, \xi\rangle} d y$ denote the Fourier transform. We consider the Bochner-Riesz means of index $\lambda$ defined by

$$
S_{t}^{\lambda} f(x)=\frac{1}{(2 \pi)^{2}} \int_{|\xi| \leq t}\left(1-\frac{|\xi|^{2}}{t^{2}}\right)^{\lambda} \widehat{f}(\xi) e^{i\langle x, \xi\rangle} d \xi
$$

and the maximal operator

$$
S_{*}^{\lambda} f(x)=\sup _{t>0}\left|S_{t}^{\lambda} f(x)\right|
$$

which controls the pointwise behavior of $S_{t}^{\lambda}$ as $t \rightarrow \infty$.

In this paper we prove weighted $L^{2}$ inequalities

$$
\int\left|S_{*}^{\lambda} f(x)\right|^{2} w(x) d x \leq C_{\lambda} \int|f(x)|^{2} W(x) d x
$$

for appropriate weights $w, W$. We shall always assume that all weights $w$ under consideration are nonnegative, locally integrable and satisfy some mild growth condition at infinity, namely

$$
\int w(x)(1+|x|)^{-N_{0}} d x<\infty
$$

for some fixed large $N_{0}$; we shall call such weights admissible.

Rubio de Francia [11] showed that for every $w \in L^{2}\left(\mathbb{R}^{2}\right)$ there is a nonnegative $W \in L^{2}\left(\mathbb{R}^{2}\right)$ such that $\|W\|_{2} \leq C_{\lambda}\|w\|_{2}, C_{\lambda}<\infty$ if $\lambda>0$, and the analogous weighted norm inequality for $S_{t}^{\lambda}$ holds uniformly in $t$. He used methods related to factorization theory of operators and the proof gave no information on how to construct $w$ from $W$. In [3] the first author explicitly constructed for every $q \geq 2$ an operator $\mathcal{W}_{q, \lambda}$, bounded on $L^{q}\left(\mathbb{R}^{2}\right)$, such that (1.1) holds for $w \in L^{q}\left(\mathbb{R}^{2}\right)$ and $W=\mathcal{W}_{q, \lambda} w$; in fact given $\mathcal{W}_{2, \lambda}$ one choses $\mathcal{W}_{q, \lambda} w$ to be $\left(\mathcal{W}_{2, \lambda}\left(w^{q / 2}\right)\right)^{2 / q}$. See also Córdoba [8] for a related result concerning $S_{t}^{\lambda}$. In [3] it was observed that the operator $\mathcal{W}_{q, \lambda}$ was bounded on $L^{r}\left(\mathbb{R}^{2}\right)$ for $q \leq r \leq 2 q$ and the question arose whether $\mathcal{W}_{q, \lambda}$ can be chosen to be independent of $q$. We shall show that this is indeed the case; for each $\lambda>0$ we construct an operator $W_{\lambda}$ such that (1.1) holds with $W=W_{\lambda}$ and $W_{\lambda}$ is bounded on $L^{r}$ if $2 \leq r \leq \infty$. Moreover this operator is pointwise bounded by a positive operator (involving a Besicovich-type maximal function acting on $w^{2}$ ) which itself is bounded on $L^{s}$ for $4 \leq s \leq \infty$.

November 25, 1997. Research supported in part by a grant from the National Science Foundation and by the International Centre for Mathematical Sciences, Edinburgh, Scotland. 
Let $\mathcal{B}_{N}$ be the family of all rectangles centered at the origin, with the property that the eccentricity (the ratio of the larger and the smaller sidelength) is equal to $2^{N}$. Define

$$
\mathfrak{M}_{N} g(x)=\sup _{\substack{R \in \mathcal{B}_{N} \\ x \in R}} \frac{1}{|R|} \int_{R}|g(x+y)| d y
$$

This maximal function is known to be bounded on $L^{2}\left(\mathbb{R}^{2}\right)$ with norm $O(N)$, moreover for $q>2$ it is bounded with norm $O\left(\frac{q}{q-2} N^{1 / 2-1 / q}\right)$ (see [7], [17]). Denote by $M$ the standard Hardy-Littlewood maximal operator and let $M_{s} g=M\left(|g|^{s}\right)^{1 / s}$.

Theorem 1. Given $\lambda>0$ there is $\delta_{\lambda}>0$ and an operator $W_{\lambda}$, bounded on $L^{q}\left(\mathbb{R}^{2}\right), 2-\delta_{\lambda} \leq q \leq \infty$, such that for all admissible weights the inequality

$$
\sup _{t>0} \int\left|S_{t}^{\lambda} f(x)\right|^{2} w(x) d x \leq C_{\lambda} \int|f(x)|^{2} W_{\lambda} w(x) d x
$$

holds. Moreover if $s>1$ then

$$
\int\left|S_{*}^{\lambda} f(x)\right|^{2} w(x) d x \leq C_{\lambda, s} \int|f(x)|^{2} M_{s}\left(W_{\lambda} w\right)(x) d x
$$

The operator $W_{\lambda}$ satisfies the pointwise estimate

$$
W_{\lambda} w(x) \leq C_{\varepsilon} \sum_{j \geq 1} 2^{-j \varepsilon}\left(\mathfrak{M}_{j / 2}\left[w^{2}\right](x)\right)^{1 / 2}, \quad \varepsilon<2 \lambda
$$

A definition of $W_{\lambda}$ and somewhat sharper results are given in $\S 2$. Stein [14, p.7] posed the question whether $W_{\lambda}$ can be essentially realized as $\sum_{l>0} 2^{-l \varepsilon} \mathfrak{M}_{l / 2} w, \varepsilon<2 \lambda$. An affirmative answer seems to be known only for radial weights (see Carbery, Romera and Soria [4]), and then only for the operator $S_{t}^{\lambda}$. Since by (1.4) the $L^{p}$ operator norm of $S_{*}^{\lambda}$ is controlled by the square root of the $L^{(p / 2)^{\prime}}$ operator norm of $M_{s} W_{\lambda}$, and since this operator is bounded for all $q \geq 2$, Theorem 1.1 implies Carbery's theorem [2] saying that $S_{*}^{\lambda}$ is bounded on $L^{p}$ if $\lambda>0$ and $2 \leq p \leq 4$. The weaker weighted norm inequality

$$
\int\left|S_{*}^{\lambda} f(x)\right|^{2} w(x) d x \leq C_{\varepsilon, s} \sum_{l>0} 2^{-l \varepsilon} \int|f(x)|^{2} M_{s}\left[\left(\mathfrak{M}_{l / 2}|w|^{2}\right)^{1 / 2}\right] d x
$$

which by (1.5) also holds true implies the known $L^{p}$ result for all $\lambda>0$ only for the range $2 \leq p \leq 8 / 3$. Moreover our estimate is interesting only for small values of $\lambda$. In fact for $\lambda>1 / 6$ M. Christ [6] showed that (1.1) holds with $W(x)=M_{s}\left[M_{r} w\right](x)$ where $r>\max \{2 /(2 \lambda+1) ; 1\}$, $s>1$.

The proof of Theorem 1.1 relies on the method used in [3]; the improvement is achieved by using arguments along the lines of [13].

In what follows $c$ and $C$ will always be positive numbers which may assume different values in different formulas. 


\section{Weighted estimates for square-functions}

Let $j>10^{5}$ and let $I$ be a fixed interval of length $2^{-j}$ contained in $[1 / 2,2]$. Let $\psi_{I} \in C_{0}^{\infty}$ be supported in $I$ and satisfy the estimates

$$
\left|\left(\frac{d}{d s}\right)^{n} \psi_{I}(s)\right| \leq C_{n} 2^{n j} .
$$

Let $\eta \in C^{\infty}\left(\mathbb{R}^{2}\right)$ supported in $\left\{\xi \in \mathbb{R}^{2}:\left|\xi_{1}\right| \leq 10^{-2} \xi_{2}\right\}$ and define

$$
\Psi(\xi)=\psi_{I}(|\xi|) \eta(\xi)
$$

and an operator $T_{t}$ by

$$
\widehat{T_{t} f}(\xi)=\Psi\left(t^{-1} \xi\right) \widehat{f}(\xi) .
$$

We are going to derive weighted $L^{2}$ inequalities for the square-functions

$$
\mathcal{G}^{k} f(x)=\left(\int_{2^{k}}^{2^{k+1}}\left|T_{t} f(x)\right|^{2} \frac{d t}{t}\right)^{1 / 2}
$$

and

$$
\mathcal{G} f(x)=\left(\int_{0}^{\infty}\left|T_{t} f(x)\right|^{2} \frac{d t}{t}\right)^{1 / 2}
$$

with suitable bounds depending on $j$.

Let $\gamma \in C_{0}^{\infty}(\mathbb{R})$ be supported in $\left(2^{-11}, 2^{11}\right)$ such that $\gamma(t)=1$ for $t \in\left(2^{-10}, 2^{10}\right)$. Let $\phi \in C_{0}^{\infty}(\mathbb{R})$ be supported in $(-1,1)$ such that

$$
\sum_{L \in \mathbb{Z}} \phi(s-L)=1, \quad s \in \mathbb{R} .
$$

For $l \leq j / 2, \tau \in \mathbb{Z},|\tau| \leq 2^{j / 2-l}$ let

$$
q_{\tau}^{j l}(\xi)=\gamma\left(2^{-l+j / 2}|\xi|\right) \phi\left(2^{-l+j / 2} \frac{\xi_{1}}{|\xi|}-\tau\right)
$$

and define for $k \in \mathbb{Z}$ an operator $Q_{\tau}^{j l, k}$ by

$$
\widehat{Q_{\tau}^{j l, k}} g(\xi)=q_{\tau}^{j l}\left(2^{-k} \xi\right) \widehat{g}(\xi) \text {. }
$$

In section 2 below we use the notation $Q_{\tau}^{j l}$ for $Q_{\tau}^{j l, 0}$.

The multipliers $q_{\tau}^{j l}\left(2^{-k}\right)$ are supported in rectangles with a longer side of length $C 2^{k+l-j / 2}$ and a shorter side of length $C 2^{k+2 l-j}$; the longer side is parallel to the radial direction $\theta(\tau)$ where $\theta(\tau)=e_{\tau} /\left|e_{\tau}\right|$ with $e_{\tau}=\left(2^{l-j / 2}, 1\right)$. The distance of these rectangles to the origin is $\approx 2^{k+l-j / 2}$.

For $\theta \in S^{1}$ let $\theta^{\perp}$ be the unit vector the vector perpendicular to $\theta$ such that $\operatorname{det}\left(\theta, \theta^{\perp}\right)=1$. Define

$$
H_{\theta, N}^{j l}(x)=2^{-l-3 j / 2}\left(1+2^{-l-j / 2}|\langle x, \theta\rangle|\right)^{-N}\left(1+2^{-j}\left|\left\langle x, \theta^{\perp}\right\rangle\right|\right)^{-N} .
$$

and for $k \in \mathbb{Z}$ the dilates

$$
H_{\theta, N}^{j l, k}(x)=2^{2 k} H_{\theta, N}^{j l}\left(2^{k} x\right) .
$$

We shall always assume that $N \geq 100+N_{0}$ where $N_{0}$ is the number in the definition of admissibility.

Now let

$$
\mathcal{W}_{j}^{k} g(x)=\sup _{\theta} H_{\theta, N}^{j 0, k} *|g|(x)
$$

and for $l>0$

$$
\mathcal{W}_{j}^{l, k} g(x)=\sup _{\tau}\left(H_{\theta(\tau), N}^{j l, k} *\left|\left(Q_{\tau}^{j l, k}\right)^{*} g\right|^{2}(x)\right)^{1 / 2}
$$


Theorem 2.1. For all $k \in \mathbb{Z}$, for all Schwartz-functions $f$, for all admissible weights $w$

$$
\int\left|\mathcal{G}^{k} f(x)\right|^{2} w(x) d x \leq C 2^{-j} \int|f(x)|^{2} W_{j}^{k} w(x) d x
$$

where

$$
W_{j}^{k} w(x)=\mathcal{W}_{j}^{k} w(x)+\sqrt{j}\left(\sum_{0<l<j / 2}\left|\mathcal{W}_{j}^{l, k} w(x)\right|^{2}\right)^{1 / 2} .
$$

Moreover if $s>1$ then

$$
\int|\mathcal{G} f(x)|^{2} w(x) d x \leq C 2^{-j} \int|f(x)|^{2} M_{s}\left[\sup _{k} W_{j}^{k} w\right](x) d x .
$$

The mapping properties of $W_{j}^{k}$ are contained in

Proposition 2.2. The inequalities

$$
\begin{aligned}
\left\|W_{j}^{k} g\right\|_{q} & \leq C(1+j)^{1-1 / q}\|g\|_{q} \\
\left\|\sup _{k} W_{j}^{k} g\right\|_{q} & \leq C(1+j)\|g\|_{q}
\end{aligned}
$$

hold for $2 \leq q \leq \infty$; here $C$ does not depend on $q, j$ or $k$. Moreover there is the pointwise estimate

$$
\sup _{k}\left|W_{j}^{k} g(x)\right| \leq C j\left(\mathfrak{M}_{j / 2}|g|^{2}\right)^{1 / 2} .
$$

We note that $(2.2),(2.4)$ and a duality argument imply the sharp $L^{4}$ estimate for the squarefunction $\mathcal{G}^{k}$, namely

$$
\left\|\mathcal{G}^{k} f\right\|_{4} \leq C(1+j)^{1 / 4} 2^{-j / 2}\|f\|_{4} .
$$

This estimate implies the known bound $\left\|T_{t}\right\|_{L^{4} \rightarrow L^{4}}=O\left(j^{1 / 4}\right)$, obtained by Córdoba [7]. The sharpness of the $L^{4}$ estimate for $\mathcal{G}^{k}$ follows from the sharpness of Córdoba's estimate. For earlier related results on $\mathcal{G}^{k}$ see [2], [3].

Remark 2.3. The estimates (2.2) and (2.3) remain true if $W_{j}^{k} w$ is replaced by

$$
W_{j, p}^{k} w=\mathcal{W}_{j}^{k} w+\sqrt{j}\left(\sum_{0<l<j / 2}\left|2^{4 l(1 / p-1 / 2)} \mathcal{W}_{j, p}^{l, k} w\right|^{2}\right)^{1 / 2}
$$

where

$$
\mathcal{W}_{j, p}^{l, k} g(x)=\sup _{\tau}\left(H_{\theta(\tau), N}^{j l, k} *\left|\left(Q_{\tau}^{j l, k}\right)^{*} g\right|^{p}(x)\right)^{1 / p}
$$

and $1 \leq p \leq 2$. The proof of this assertion will be given below.

Standard arguments ([15], [3], [16]) relating maximal operators to square functions can be used to deduce Theorem 1 from the above results. Namely let $\widetilde{\Psi}(\xi)=\langle\xi, \nabla \Psi(\xi)\rangle$ and and let $\widetilde{T}_{t}$ be the convolution operator with Fourier multiplier $\widetilde{\Psi}\left(t^{-1} \cdot\right)$; then $2^{-j} \widetilde{T}_{t}$ satisfies the same quantitative properties as $T_{t}$. Using [16, p.499] one obtains the estimate

$$
\begin{aligned}
\sup _{t>0}\left|T_{t} f(x)\right| & \leq\left(\sum_{k \in \mathbb{Z}^{1}} \sup _{1 \leq 2}\left|T_{2^{k_{s}}} f\right|^{2}\right)^{1 / 2} \\
& \leq\left(\sum_{k \in \mathbb{Z}}\left[2^{j / 2}\left(\int_{1}^{2}\left|T_{2^{k_{s}}} f\right|^{2} d s\right)^{1 / 2}+2^{-j / 2}\left(\int_{1}^{2}\left|\frac{d}{d s} T_{2^{k_{s}}} f\right|^{2} d s\right)^{1 / 2}\right]^{2}\right)^{1 / 2} \\
& \leq C\left[2^{j / 2}\left(\int_{0}^{\infty}\left|T_{t} f\right|^{2} \frac{d t}{t}\right)^{1 / 2}+2^{-j / 2}\left(\int_{0}^{\infty}\left|\widetilde{T}_{t} f\right|^{2} \frac{d t}{t}\right)^{1 / 2}\right]
\end{aligned}
$$


and therefore

$$
\int \sup _{t>0}\left|T_{t} f(x)\right|^{2} w(x) d x \leq C \int|f(x)|^{2} M_{s}\left(\sup _{k} W_{j}^{k} w\right)(x) d x .
$$

Now $S_{t}^{\lambda}=\sum_{j=0}^{\infty} 2^{-j \lambda} S_{j, t}$ where $\sup _{t>0}\left|S_{j, t} f\right|$ is pointwise bounded by $2^{j} M f$ and where for $j \geq 10^{5}$ the Fourier multiplier for $S_{j, t}$ is $C \psi_{I}\left(t^{-1}\right.$.) with a suitable $\psi_{I}$ satisfying the bounds (2.1). Therefore Theorem 2.1, Remark 2.3 and a weighted inequality for $M$ due to Fefferman and Stein ([16, p. 53]) imply that (1.1) holds with $W=M_{s}\left(W_{\varepsilon, p} w\right), s>1$, where

$$
W_{\varepsilon, p} w(x)=M w(x)+\sum_{j \geq 10^{5}} 2^{-j \varepsilon} \sup _{k} \widetilde{W}_{j, p}^{k} w(x) .
$$

Here $1 \leq p \leq 2, \varepsilon<2 \lambda$ and $\widetilde{W}_{j, p}^{k} w$ is a sum of less than $10^{6}$ operators satisfying the same quantitative estimates as the operator $W_{j, p}^{k}$ in Remark 2.3 (they are essentially rotates of this operator). By Proposition 2.2 the operator $W_{\varepsilon, 2}$ is bounded on $L^{q}$, for $2<q \leq \infty$. An examination of the operators $\widetilde{W}_{j, p}^{k}$ and an interpolation argument show that for $1<p<2$

$$
\left\|\sup _{k} \widetilde{W}_{j, p}^{k} w\right\|_{p} \leq C 2^{j a(1-2 / p)}\|w\|_{p}
$$

for some $a>0$. This implies that given $\varepsilon>0$ there is $p<2$ and $\delta(\varepsilon, p)>0$ such that $W_{\varepsilon, p}$ is bounded on $L^{q}$ for $2-\delta(\varepsilon, p) \leq q \leq \infty$. Theorem 1 follows by choosing $0<\varepsilon<2 \lambda$.

Before proceeding with the proof of Theorem 2.1 we state without proof a lemma which is closely related to a theorem of Carleson concerning square-functions with equally spaced decompositions. For published proofs see [7], [12] (and also [13] for a simple proof based on Bernstein's Theorem).

Lemma 2.4. Let $\left\{Q_{l}\right\}$ be a sequence of disjoint unit cubes and let $m_{l}$ be supported in $Q_{l}$; moreover assume that the estimates

$$
\int\left|\partial_{\xi}^{\alpha} m_{l}(\xi)\right|^{2} d \xi \leq B^{2}
$$

hold for all multiindices $|\alpha| \leq N$, uniformly in l. Let $A \in G L(2, \mathbb{R})$. Then

$$
\left.\sum_{l} \mid \mathcal{F}^{-1}\left[m_{l}(A \cdot) \mathcal{F} f\right](x)\right]\left.\right|^{2} \leq C_{N} B^{2} \int|f(y)|^{2} \frac{|\operatorname{det} A|^{-1}}{1+\left|{ }^{t} A^{-1}(x-y)\right|^{2 N}} d y .
$$

We now fix $j \geq 10$. In what follows we shall introduce various decompositions depending on $j$ without always indicating the dependence on $j$. Consequently we shall also omit the index $j$ in $H_{\tau, N}^{j k l}$ or $Q_{\tau}^{j k l}$. Various constants $C$ in inequalities may depend on $N$.

Proof of Theorem 2.1. Denote by $\delta_{k}$ the dilation operator given by $\delta_{k} f(x)=f\left(2^{-k} x\right)$. Then $\mathcal{G}_{k}=\delta_{-k} \mathcal{G}_{0} \delta_{k}$ and $W_{j}^{k}=\delta_{-k} W_{j}^{0} \delta_{k}$. A scaling argument shows that in order to prove (2.2) it suffices to prove (2.2) for $k=0$ which is henceforth assumed.

For $m \in \mathbb{Z}$, define operators $P^{m}$ by

$$
\widehat{P^{m}} f(\xi)=\phi\left(2^{j}|\xi|-m\right) \widehat{f}(\xi)
$$

so that the $\widehat{P^{m}} f$ are supported in thin annuli of width $2^{-m+1}$ and $\sum P^{m} f=f$. Observe that for fixed $t$ there are at most three $m$ such that $P^{m} T_{t} \neq 0$. Therefore

$$
\begin{aligned}
\int\left|\mathcal{G}^{0} f(x)\right|^{2} w(x) d x & =\iint_{\mathbb{R}^{2} \times[1,2]}\left|\sum_{m} P^{m} T_{t} f(x)\right|^{2} w(x) d x \frac{d t}{t} \\
& \leq C \iint_{\mathbb{R}^{2} \times[1,2]} \sum_{m}\left|P^{m} T_{t} f(x)\right|^{2} w(x) d x \frac{d t}{t} .
\end{aligned}
$$


Define for $\nu=\left(\nu_{1}, \nu_{2}\right) \in \mathbb{Z}^{2},\left|\nu_{1}\right|,\left|\nu_{2}\right| \leq 2^{j / 2}$

$$
\begin{aligned}
& \widehat{P_{\nu} f}(\xi)=\phi\left(2^{j / 2} \xi_{1}-\nu_{1}\right) \phi\left(2^{j / 2} \xi_{2}-\nu_{2}\right) \widehat{f}(\xi) \\
& \widehat{P_{\nu}^{m} f}(\xi)=\phi\left(2^{j / 2} \xi_{1}-\nu_{1}\right) \phi\left(2^{j / 2} \xi_{2}-\nu_{2}\right) \widehat{P^{m}} f(\xi) .
\end{aligned}
$$

Thus $\widehat{P_{\nu} f}$ is supported in a square of sidelength $2^{1-j / 2}$, and $\widehat{P_{\nu}^{m} f}$ is supported in the intersection of such a square with a thin annulus of width $2^{1-j}$; therefore it is supported in a rectangle of dimensions $C 2^{-j / 2} \times C 2^{-j}$.

Moreover for fixed $l$ and for $\mu \in \mathbb{Z}$ we define operators $P_{\nu \mu}^{m l}$ by

$$
\widehat{P_{\nu \mu}^{m l} f}(\xi)=\phi\left(2^{l+j / 2} \xi_{1}-\mu\right) \widehat{P_{\nu}^{m} f}(\xi) .
$$

so that $\widehat{P_{\nu \mu}^{m l} f}$ is supported in a smaller rectangle of dimensions $C 2^{-l-j / 2} \times C 2^{-j}$.

Finally define operators $B_{\tau \sigma \rho}^{l}$ by

$$
\widehat{B_{\tau \sigma \rho}^{l} f}(\xi)=b_{\tau \sigma \rho}^{l}(\xi) \widehat{f}(\xi)
$$

where

$$
b_{\tau \sigma \rho}^{l}(\xi)=q_{\tau}^{j l}(\xi) \phi\left(2^{l+j / 2}\langle\xi, \theta(\tau)\rangle-\rho\right) \phi\left(2^{j}\left\langle\xi, \theta^{\perp}(\tau)\right\rangle-\sigma\right)
$$

here $q_{\tau}^{j l}$ and the corresponding operator $Q_{\tau}^{j l}$ were defined above.

Let

$$
\mathcal{Z}_{l}=\left\{\left(\nu, \nu^{\prime}\right) \in \mathbb{Z}^{2} \times \mathbb{Z}^{2}: 2^{l} \leq\left|\nu_{1}-\nu_{1}^{\prime}\right|<2^{l+1}\right\}
$$

and for $\kappa \in \mathbb{Z},|\kappa| \leq 2^{j / 2-\ell}$ let

$$
\begin{aligned}
\mathfrak{Z}_{l \kappa} & =\left\{\nu: 2^{l}(\kappa-1)<\nu \leq 2^{l} \kappa\right\} . \\
\mathfrak{A}_{l \kappa} & =\left\{\mu: 2^{2 l}(\kappa-4) \leq \mu \leq 2^{2 l}(\kappa+4)\right\}
\end{aligned}
$$

We shall use the following elementary geometrical facts (2.7-2.15), assuming $l \geq 10$ in what follows.

(2.7) For each $m, \nu_{1}$ there are at most three $\nu_{2}$ such that $P_{\nu}^{m} \neq 0\left(\right.$ here $\left.\nu=\left(\nu_{1}, \nu_{2}\right)\right)$.

(2.8) For each $\mu$ there are at most nine $\nu$ such that $P_{\nu \mu}^{m l} \neq 0$.

(2.9) If $\nu \in \mathfrak{Z}_{l \kappa},\left(\nu, \nu^{\prime}\right) \in \mathcal{Z}_{l}$ then $\nu^{\prime} \in \mathfrak{Z}_{l \kappa^{\prime}}$ with $\left|\kappa-\kappa^{\prime}\right| \leq 1$.

(2.10) If $\nu \in \mathfrak{Z}_{l \kappa^{\prime}}$, with $\left|\kappa-\kappa^{\prime}\right| \leq 1$ and if $P_{\nu \mu}^{m l} \neq 0$ then $\mu \in \mathfrak{A}_{l \kappa}$.

(2.11) For each $\mu \in \mathfrak{A}_{l \kappa}$ the support of $\widehat{P_{\nu \mu}^{m l} f}$ is contained in a rectangle $R_{\mu}^{l}$ with sidelengths $C_{1} 2^{-l-j / 2}$ and $C_{1} 2^{-j}$ where the orientation of $R_{\mu}^{l}$ only depends on $\kappa$; the longer side is parallel to $u_{\kappa}=\left(-1,2^{l-j / 2} \kappa\right)$. The rectangle is contained in the annulus $\left\{\xi:|| \xi\left|-2^{-j} m\right| \leq C 2^{-j}\right\}$ (here $\left.2^{-j} m \in[1 / 2,2]\right)$. The differentiability properties of the multiplier corresponding to $P_{\nu \mu}^{m l}$ satisfy the same bounds as a bump function adapted to $R_{\mu}^{l}$. 
(2.12) For each $\mu, \mu^{\prime}$ the set

$$
\operatorname{supp}\left(\widehat{P_{\nu \mu}^{l} f}\right)-\operatorname{supp}\left(\widehat{P_{\nu^{\prime} \mu^{\prime}}^{l} f}\right)
$$

is contained in a rectangle $\widetilde{R}_{\mu \mu^{\prime}}^{l}$ with sidelengths $C_{2} 2^{-l-j / 2}$ and $C_{2} 2^{-j}$. The rectangle $\widetilde{R}_{\mu \mu^{\prime}}^{l}$ is contained in an annulus $\left\{\xi: c_{3} 2^{l-j / 2} \leq|\xi| \leq C_{3} 2^{l-j / 2}\right\}$. The longer side is parallel to $u_{\kappa}=$ $\left(-1,2^{l-j / 2} \kappa\right)$.

(2.13) Fix $m, l$. Then there is a constant $C_{4}$, independent of $l, m$ such that each $\xi \in \mathbb{R}^{2}$ is contained in at most $C_{4}$ of the sets supp $\left(\widehat{P_{\nu \mu}^{m l} f}\right)-\operatorname{supp}\left(\widehat{P_{\nu^{\prime} \mu^{\prime}}^{m l} f}\right)$.

$$
\begin{aligned}
& \text { Let } p_{\mu}^{m l}(\xi)=\phi\left(2^{l+j / 2} \xi_{1}-\mu\right) \phi\left(2^{j}|\xi|-m\right) \text {. Let } \\
& \qquad \mathfrak{S}_{\mu \mu^{\prime}}^{m l \tau}=\left\{(\sigma, \rho):\left(\operatorname{supp} p_{\mu}^{m l}-\operatorname{supp} p_{\mu^{\prime}}^{m l}\right) \cap \operatorname{supp} b_{\tau \sigma \rho}^{l} \neq \emptyset\right\}
\end{aligned}
$$

and $\mathfrak{S}_{\mu \mu^{\prime}}^{m l}=\cup_{\tau} \mathfrak{S}_{\mu \mu^{\prime}}^{m l \tau}$. Then the cardinality of $\mathfrak{S}_{\mu \mu^{\prime}}^{m l}$ is bounded, independently of $m, l, \mu, \mu^{\prime}$. Likewise, if

$$
\mathfrak{R}_{\tau \sigma \rho}^{m l \kappa}=\left\{\left(\mu, \mu^{\prime}\right) \in \mathfrak{A}_{l \kappa} \times \mathfrak{A}_{l \kappa}:\left(\operatorname{supp} p_{\mu}^{m l}-\operatorname{supp} p_{\mu^{\prime}}^{m l}\right) \cap \operatorname{supp} b_{\tau \sigma \rho}^{l} \neq \emptyset \text { for some } \tau\right\}
$$

and if $\mathfrak{R}_{\tau \sigma \rho}^{m l}=\cup_{\kappa} \mathfrak{R}_{\tau \sigma \rho}^{m l \kappa}$ then the cardinality of $\mathfrak{R}_{\tau \sigma \rho}^{m l}$ is bounded, independently of $m, l, \sigma, \rho$.

(2.15) The cardinality of the set

$$
\mathfrak{T}_{\kappa}^{l}=\left\{\tau: \mathfrak{R}_{\tau \sigma \rho}^{m l \kappa} \neq \emptyset \text { for some }(\sigma, \rho, m)\right\}
$$

is bounded, independently of $l$.

For fixed $m$ we now write

$$
\begin{aligned}
& \iint\left|P^{m} T_{t} f(x)\right|^{2} w(x) d x \frac{d t}{t} \\
= & \iint\left|\sum_{\nu} P_{\nu}^{m} T_{t} f(x)\right|^{2} w(x) d x \frac{d t}{t} \\
= & \iint \sum_{\nu, \nu^{\prime}} P_{\nu}^{m} T_{t} f(x) \overline{P_{\nu^{\prime}}^{m} T_{t} f(x)} w(x) d x \frac{d t}{t} .
\end{aligned}
$$

Then

$$
\iint \sum_{\nu, \nu^{\prime}} P_{\nu}^{m} T_{t} f(x) \overline{P_{\nu^{\prime}}^{m} T_{t} f(x)} w(x) d x \frac{d t}{t} \leq C\left[J^{m}+\sum_{10 \leq l \leq j / 2}\left|I_{l}^{m}\right|\right]
$$

where

$$
J^{m}=\iint \sum_{\nu}\left|P_{\nu}^{m} T_{t} f(x)\right|^{2} w(x) d x \frac{d t}{t}
$$

and

$$
I_{l}^{m}=\iint \sum_{\left(\nu, \nu^{\prime}\right) \in \mathcal{Z}_{l}} P_{\nu}^{m} T_{t} f(x) \overline{P_{\nu^{\prime}}^{m} T_{t} f(x)} w(x) d x \frac{d t}{t}
$$


By (2.9)

$$
I_{l}^{m}=\iint \sum_{\substack{\left(\kappa, \kappa^{\prime}\right) \\\left|\kappa-\kappa^{\prime}\right| \leq 1}} \sum_{\substack{\left(\nu, \nu^{\prime}\right) \in \mathcal{Z}_{l} \\ \nu \in \mathfrak{Z}_{l \kappa}}} P_{\nu}^{m} T_{t} f(x) \overline{P_{\nu^{\prime}}^{m} T_{t} f(x)} w(x) d x \frac{d t}{t} .
$$

Therefore by $(2.10)$

$$
I_{l}^{m}=\sum_{\kappa} I_{l \kappa}^{m}
$$

where

$$
I_{l \kappa}^{m}=\iint \sum_{\substack{\left(\nu, \nu^{\prime}\right) \in \mathcal{Z}_{l} \\ \nu \in \mathfrak{Z}_{l \kappa}}} \sum_{\substack{\left(\mu, \mu^{\prime}\right) \in \\ \mathfrak{A}_{l \kappa} \times \mathfrak{A}_{l \kappa}}} P_{\nu \mu}^{m l} T_{t} f(x) \overline{P_{\nu^{\prime} \mu^{\prime}}^{m l} T_{t} f(x)} w(x) d x \frac{d t}{t} .
$$

Now we can write

$$
I_{l \kappa}^{m}=\iint \sum_{\tau, \rho, \sigma} \sum_{\substack{\left(\nu, \nu^{\prime}\right) \in \mathcal{Z}_{l} \\ \nu \in \mathfrak{Z}_{l \kappa}}} \sum_{\substack{\left(\mu, \mu^{\prime}\right) \in \\ \mathfrak{A}_{l \kappa} \times \mathfrak{A}_{l \kappa}}} B_{\tau \sigma \rho}^{l}\left[P_{\nu \mu}^{k m l} T_{t} f \overline{P_{\nu^{\prime} \mu^{\prime}}^{k m l} T_{t} f}\right](x) w(x) d x \frac{d t}{t}
$$

and we obtain using (2.7), (2.8), (2.14) and (2.15) together with various applications of the CauchySchwarz inequality

$$
\begin{aligned}
& I_{l \kappa}^{m} \leq \sum_{\tau \in \mathfrak{T}_{\kappa}^{l}} \iint \sum_{\substack{\left(\nu, \nu^{\prime}\right) \in \mathcal{Z}_{l} \\
\nu \in \mathfrak{Z}_{l \kappa}}} \sum_{\substack{\left(\mu, \mu^{\prime}\right) \in \\
\mathfrak{A}_{l \kappa} \times \mathfrak{A}_{l \kappa}}} P_{\nu \mu}^{m l} T_{t} f(x) \overline{P_{\nu^{\prime} \mu^{\prime}}^{m l} T_{t} f(x)} \sum_{\substack{(\sigma, \rho) \\
\in \mathfrak{S}_{\mu \mu^{\prime}}^{m l}}}\left(B_{\tau \sigma \rho}^{l}\right)^{*} w(x) d x \frac{d t}{t} \\
& \leq \iint\left(\sum_{\substack{\left(\mu, \mu^{\prime}\right) \in \\
\mathfrak{A}_{l \kappa} \times \mathfrak{A}_{l \kappa}}}\left|\sum_{\substack{\left(\nu, \nu^{\prime}\right) \in \mathcal{Z}_{l} \\
\nu \in \mathfrak{Z}_{l \kappa}}} P_{\nu \mu}^{m l} T_{t} f(x) \overline{P_{\nu^{\prime} \mu^{\prime}}^{m l} T_{t} f(x)}\right|^{2}\right)^{1 / 2} \\
& \times\left(\sum_{\substack{\left(\mu, \mu^{\prime}\right) \in \\
\mathfrak{A}_{l \kappa} \times \mathfrak{A}_{l \kappa}}}\left|\sum_{\tau \in \mathfrak{T}_{\kappa}^{l}} \sum_{\substack{(\sigma, \rho) \\
\in \mathfrak{S}_{\mu \mu^{\prime}}^{m l}}}\left(B_{\tau \sigma \rho}^{l}\right)^{*} w(x)\right|^{2}\right)^{1 / 2} d x \frac{d t}{t} \\
& \leq C \iint\left(\left.\sum_{\substack{\left(\mu, \mu^{\prime}\right) \in \\
\mathfrak{A}_{l \kappa} \times \mathfrak{A}_{l \kappa}}}\left|\sum_{\substack{\left(\nu, \nu^{\prime}\right) \in \mathcal{Z}_{l} \\
\nu \in \mathfrak{Z}_{l \kappa}}}\right| P_{\nu \mu}^{m l} T_{t} f(x) \overline{P_{\nu^{\prime} \mu^{\prime}}^{m l} T_{t} f(x)}\right|^{2}\right)^{1 / 2} \\
& \times\left(\sum_{\tau \in \mathfrak{T}_{\kappa}^{l}} \sum_{\sigma, \rho} \sum_{\substack{\left(\mu, \mu^{\prime}\right) \\
\in \mathfrak{R}_{\tau \sigma \rho}^{m l}}}\left|\left(B_{\tau \sigma \rho}^{l}\right)^{*} w(x)\right|^{2}\right)^{1 / 2} d x \frac{d t}{t} \\
& \leq C \iint \sum_{\nu \in \mathfrak{Z}_{l \kappa}} \sum_{\mu \in \mathfrak{A}_{l \kappa}}\left|P_{\nu \mu}^{m l} T_{t} f\right|^{2} \sup _{\tau \in \mathfrak{T}_{\kappa}^{l}}\left(\sum_{\sigma, \rho}\left|\left(B_{\tau \sigma \rho}^{l}\right)^{*} w(x)\right|^{2}\right)^{1 / 2} d x \frac{d t}{t} .
\end{aligned}
$$

Now an application of Lemma 2.4 yields

$$
\left(\sum_{\sigma, \rho}\left|\left(B_{\tau \sigma \rho}^{l}\right)^{*} w(x)\right|^{2}\right)^{1 / 2} \leq C\left(H_{\tau, 4 N}^{l} *\left|\left(Q_{\tau}^{j l}\right)^{*} w\right|^{2}(x)\right)^{1 / 2}
$$

We summarize: 


\section{Lemma 2.5.}

$$
\int\left|\mathcal{G}^{0} f(x)\right|^{2} w(x) d x \leq C \sum_{m} J^{m}+C \sum_{m} \sum_{10 \leq l \leq j / 2}\left|I_{l}^{m}\right|
$$

where

$$
J^{m}=\int \sum_{\nu} \int\left|P_{\nu}^{m} T_{t} f(x)\right|^{2} \frac{d t}{t} w(x) d x
$$

and

$$
\left|I_{l}^{m}\right| \leq C \int \sum_{\kappa} \sum_{\nu \in \mathfrak{Z}_{l \kappa}} \sum_{\mu \in \mathfrak{A}_{l \kappa}} \int\left|P_{\nu \mu}^{m l} T_{t} f(x)\right|^{2} \frac{d t}{t} \sup _{\tau \in \mathfrak{T}_{\kappa}^{l}}\left(H_{\tau, 4 N}^{l} *\left|\left(Q_{\tau}^{j l}\right)^{*} w\right|^{2}(x)\right)^{1 / 2} d x
$$

We continue with the proof of Theorem 2.1. One checks by a straightforward integration by parts using (2.11) that if $\mu \in \mathfrak{A}_{l \kappa}$ and $\tau \in \mathfrak{T}_{\kappa}^{l}$ then

$$
\left|\mathcal{F}^{-1}\left[\psi_{I}\left(t^{-1} \cdot\right) p_{\mu}^{m l}\right](x)\right| \leq C H_{\tau, 4 N}^{k l}(x),
$$

here $N \gg 2$ and $p_{\mu}^{m l}$ was defined in (2.14). Moreover the $t^{-1} d t$ measure of the set $\left\{t: P^{m} T_{t} \neq 0\right\}$ is bounded by $c 2^{-j}$. Also observe that for $\tau \in \mathfrak{T}_{\kappa}^{l}$ the kernels $H_{\tau^{\prime}, 4 N}^{l}$ behave essentially the same; in fact

$$
\sup _{\tau^{\prime} \in \mathfrak{T}_{\kappa}^{l}} H_{\tau^{\prime}, 4 N}^{l}(x) \leq C \inf _{\tau \in \mathfrak{T}_{\kappa}^{l}} H_{\tau, 4 N}^{l}(x)
$$

An application of Lemma 2.4 yields

$$
\int \sum_{m} \sum_{\nu \in \mathfrak{Z}_{l \kappa}} \sum_{\mu \in \mathfrak{A}_{l \kappa}} \int\left|P_{\nu \mu}^{m l} T_{t} f(x)\right|^{2} \frac{d t}{t} w(x) d x \leq C 2^{-j} \int \sum_{\nu \in \mathfrak{Z}_{l \kappa}}\left|P_{\nu} f(x)\right|^{2} H_{\tau, 3 N}^{l} * w(x) d x
$$

for all $\tau \in \mathfrak{T}_{\kappa}^{l}$.

Next, the convolution kernel of $P_{\nu}$ is pointwise bounded by

$$
\widetilde{H}_{j / 2,3 N}(x)=2^{2(j / 2)}\left(1+2^{j / 2}|x|\right)^{-3 N}
$$

and by another application of Lemma 2.4 we obtain the inequality

$$
\int \sum_{\kappa} \sum_{\nu \in \mathfrak{Z}_{l \kappa}}\left|P_{\nu} f(x)\right|^{2} w(x) d x \leq C \int|f(x)|^{2} \widetilde{H}_{j / 2,3 N} * w(x) d x .
$$

Since for large $N$

$$
H_{\tau, 4 N}^{l} * \widetilde{H}_{j / 2,4 N}(x) \leq C H_{\tau, 3 N}^{l}(x)
$$

we obtain from $(2.18),(2.19),(2.20)$ and Lemma 2.5 the estimate

$$
\begin{aligned}
& \sum_{10 \leq l \leq \frac{j}{2}} \sum_{m} I_{l}^{m} \\
& \leq C 2^{-j} \int|f(x)|^{2} \sum_{10 \leq l \leq \frac{j}{2}} \sup _{\substack{2^{l-j / 2} \tau \\
\in\left[16^{-1}, 16\right]}} H_{\tau, 3 N}^{l} *\left(H_{\tau, 3 N}^{l} *\left|\left(Q_{\tau}^{j l}\right)^{*} w(y)\right|^{2}\right)^{1 / 2}(x) d x \\
& \leq C 2^{-j} \int|f(x)|^{2} \sqrt{j}\left(\sum_{10 \leq l \leq \frac{j}{2}}\left|\mathcal{W}_{j} w\right|^{2}\right)^{1 / 2} d x
\end{aligned}
$$


Similarly

$$
\sum_{m} J^{m} \leq C 2^{-j} \int|f(x)|^{2} \sup _{\theta} H_{\theta, N}^{0} * w(x) d x
$$

and we obtain $(2.2)$.

Finally let $\beta \in C_{0}^{\infty}\left(\mathbb{R}^{2}\right)$ such that $\beta(\xi)=1$ if $1 / 4 \leq|\xi| \leq 4$ and $\beta(\xi)=0$ if $|\xi| \notin(1 / 8,8)$ and define the Littlewood-Paley operator $L^{k}$ by

$$
\widehat{L^{k}} f(\xi)=\beta\left(2^{-k} \xi\right) \widehat{f}(\xi)
$$

Observe that

$$
\mathcal{G}^{k} f=\mathcal{G}^{k}\left(L^{k-1} f+L^{k} f+L^{k+1} f\right) .
$$

Now $f \mapsto\left\{L^{k} f\right\}$ defines a vector-valued regular singular integral operator and there is the CórdobaFefferman weighted norm inequality

$$
\int \sum_{k}\left|L^{k} f(x)\right|^{2} w(x) d x \leq C_{s} \int|f(x)|^{2} M_{s} w(x) d x, \quad s>1
$$

see [10]. Consequently (2.2) yields also (2.3).

Proof of Remark 2.3. This requires a modification of (2.17). Let

$$
\widehat{\Gamma_{\tau \rho \sigma}^{l} f}(\xi)=\phi\left(2^{l+j / 2}\langle\xi, \theta(\tau)\rangle-\rho\right) \phi\left(2^{j}\left\langle\xi, \theta^{\perp}(\tau)\right\rangle-\sigma\right) \widehat{f}(\xi)
$$

then by definition $\Gamma_{\tau \rho \sigma}^{l} Q_{\tau}^{j l}=B_{\tau \sigma \rho}^{l}$. Now let $\mathfrak{U}_{\tau}^{l}$ be the set of all pairs $(\rho, \sigma)$ such that $\Gamma_{\tau \rho \sigma}^{l} Q_{\tau}^{j l} \neq 0$. Then the cardinality of $\mathfrak{U}_{\tau}^{l}$ is bounded by $C 2^{4 l}$ where $C$ does not depend on $j$ or $\tau$. Since the convolution kernel of $\Gamma_{\tau \rho \sigma}^{l} Q_{\tau}^{j l}$ is bounded by $C H_{\tau, N}^{l}$ we obtain

$$
\left(\sum_{(\rho, \sigma) \in \mathfrak{U}_{\tau}^{l}}\left|\Gamma_{\tau \rho \sigma}^{l} g(x)\right|^{2}\right)^{1 / 2} \leq C 2^{2 l} H_{\tau, N}^{l} *|g|(x) .
$$

An application of Lemma 2.4 and an interpolation argument then show that (2.17) can be replaced by

$$
\left(\sum_{\sigma, \rho}\left|\left(B_{\tau \sigma \rho}^{l}\right)^{*} w(x)\right|^{2}\right)^{1 / 2} \leq C 2^{4 l(1 / p-1 / 2)}\left(H_{\tau, N}^{l} *\left|Q_{\tau}^{j l} w\right|^{p}(x)\right)^{1 / p}
$$

if $1 \leq p \leq 2$. The rest of the proof requires only notational changes.

Proof of Proposition 2.2. The convolution kernel of $Q_{\tau}^{j l, k}$ is bounded by a constant $C_{N}$ times

$$
\mathcal{H}_{\theta(\tau), N}^{l, k}(x)=2^{2 k+3 l-3 j / 2}\left(1+2^{k+l-j / 2}|\langle x, \theta(\tau)\rangle|\right)^{-N}\left(1+2^{k+2 l-j}\left|\left\langle x, \theta(\tau)^{\perp}\right\rangle\right|\right)^{-N}
$$

Moreover a straightforward calculation shows that

$$
H_{\theta, N}^{l, k} *\left|\mathcal{H}_{\theta, N}^{l, k} * w\right|^{2} \leq C \mathfrak{M}_{j / 2}\left[w^{2}\right]
$$


pointwise which implies (2.5). For the same reason $\mathcal{W}_{j}^{k l}$ is uniformly bounded on $L^{\infty}$ and the analogue of the inequality

$$
\left\|\left(\sum_{0<l<j / 2}\left|\mathcal{W}_{j}^{l, k} g\right|^{2}\right)^{1 / 2}\right\|_{q} \leq C j^{1 / 2-1 / q}\|g\|_{q}
$$

holds for $q=\infty$. On $L^{2}$ we use an orthogonality argument. Since for fixed $k, j$ each $\xi \in R^{2}$ is contained in only a bounded number of the sets supp $\widehat{Q_{\tau}^{j l, k}} g,\left(0<l<j / 2,|\tau| \leq 2^{j / 2-l}\right)$ we obtain

$$
\begin{aligned}
\left\|\left(\sum_{0<l<j / 2}\left|\mathcal{W}_{j}^{l, k} g\right|^{2}\right)^{1 / 2}\right\|_{2} & \leq C\left\|\left(\sum_{0<l<j / 2} \sum_{\tau} H_{\theta(\tau), N}^{l, k} *\left|\left(Q_{\tau}^{j l, k}\right)^{*} g\right|^{2}\right)^{1 / 2}\right\|_{2} \\
& \leq C\left(\sum_{0<l<j / 2} \sum_{\tau}\left\|\left(Q_{\tau}^{j l, k}\right)^{*} g\right\|_{2}^{2}\right)^{1 / 2} \leq C^{\prime}\|g\|_{2} .
\end{aligned}
$$

The desired bound (2.22) follows by interpolation.

In order to estimate the maximal function $\sup _{k} W_{j}^{k} g$ in $L^{q}$ we dominate the sup by an $\ell^{q}$ norm and the asserted inequality follows from

$$
\left(\sum_{k}\left\|\left(\sum_{0<l<j / 2}\left|\mathcal{W}_{j}^{l, k} g\right|^{2}\right)^{1 / 2}\right\|_{q}^{q}\right)^{1 / q} \leq C(1+j)^{1 / 2}\|g\|_{q}
$$

Now the analogue of (2.23) for $q=\infty$ follows as before. For $q=2$ we use the observation that for fixed $j, k$ the functions $q_{\tau}^{j l}\left(2^{-k}\right.$.) are supported in an annulus $\left\{\xi: c_{0} 2^{k-j / 2} \leq|\xi| \leq c_{1} 2^{k}\right\}$. Therefore the above $L^{2}$ argument yields now an additional factor of $\sqrt{1+j}$, proving (2.23) for $q=2$.

\section{REFERENCES}

1. L. Carleson and P. Sjölin, Oscillatory integrals and a multiplier problem for the disc, Studia Math. 44 (1972), 287-299.

2. A. Carbery, The boundedness of the maximal Bochner-Riesz operator on $L^{4}\left(R^{2}\right)$, Duke Math. J. 50 (1983), 409-416.

3. (1985), 673-679.

4. A. Carbery, E. Romera and F. Soria, Radial weights and mixed norm inequalities for the disc multiplier, J. Funct. Anal. 109 (1992), 52-75.

5. A. Carbery and A. Seeger, Homogeneous Fourier multipliers of Marcinkiewicz type, Ark. Mat. 33 (1995), 45-80.

6. M. Christ, On the almost everywhere convergence of Bochner-Riesz means in higher dimensions, Proc. Amer. Math. Soc. 95 (1985), 16-20.

7. A. Córdoba, A note on Bochner-Riesz operators, Duke Math. J. 46 (1979), 505-511.

8. _ An integral inequality for the disc multiplier, Proc. Amer. Math. Soc. 92 (1984), 407-408.

9. C. Fefferman, A note on spherical summation multipliers, Israel J. Math. 15 (1973), 44-52.

10. J. García-Cuerva and J. L. Rubio de Francia, Weighted norm inequalities and related topics, North-Holland Math. Studies 116, North-Holland, 1985.

11. J. L. Rubio de Francia, Weighted norm inequalities and vector valued inequalities, Proc. Conf. Harmonic Analysis (Minneapolis 1981), ed. by F. Ricci and G. Weiss, Lecture notes in Math., vol. 908, Springer-Verlag, Berlin, New York, 1982, pp. 86-101.

12. __ Estimates for some square functions of Littlewood-Paley type, Publicacions Mathemàtiques 27 (1983), 81-108.

13. A. Seeger, Endpoint inequalities for Bochner-Riesz multipliers in the plane, Pacific J. Math. 174 (1996), 543553. 
14. E. M. Stein, Some problems in harmonic analysis, Proc. Symp. Pure Math., vol. 35,I, American Mathematical Society, Providence, R.I., 1979, pp. 3-20.

15. E. M. Stein and G. Weiss, Introduction to Fourier analysis on Euclidean spaces, Princeton Univ. Press, Princeton, N.J., 1971.

16. E.M. Stein, Harmonic analysis: Real variable methods, orthogonality and oscillatory integrals, Princeton Univ. Press, 1993.

17. J.-O. Strömberg, Maximal functions associated to rectangles with uniformly distributed directions, Annals Math. 107 (1978), 399-402.

Department of Mathematics and Statistics, University of Edinburgh, King's Buildings, Mayfield RD., Edinburgh EH3 9JZ

Department of Mathematics, University of Wisconsin, Madison, Wi 53706 